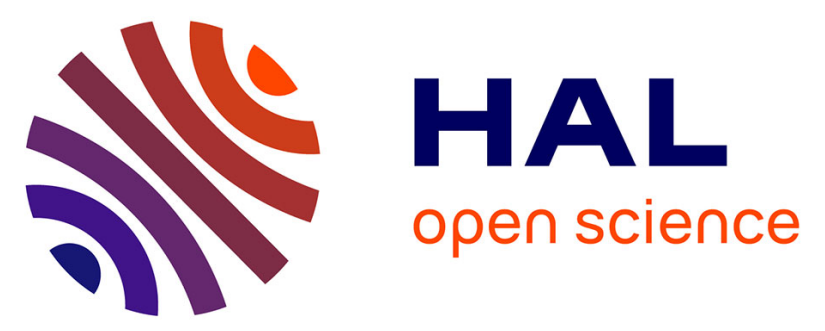

\title{
Force localized interaction sensing system for HYDRÖ̈D humanoid robot
}

Mohamed Tahoun, Ahmad Tayba, Samer Alfayad, Abdelmoneim Wahdan, Luc Chassagne

\section{- To cite this version:}

Mohamed Tahoun, Ahmad Tayba, Samer Alfayad, Abdelmoneim Wahdan, Luc Chassagne. Force localized interaction sensing system for HYDROïD humanoid robot. IEEE Sensors Journal, 2020. hal-03081642

\section{HAL Id: hal-03081642 \\ https://hal.science/hal-03081642}

Submitted on 18 Dec 2020

HAL is a multi-disciplinary open access archive for the deposit and dissemination of scientific research documents, whether they are published or not. The documents may come from teaching and research institutions in France or abroad, or from public or private research centers.
L'archive ouverte pluridisciplinaire HAL, est destinée au dépôt et à la diffusion de documents scientifiques de niveau recherche, publiés ou non, émanant des établissements d'enseignement et de recherche français ou étrangers, des laboratoires publics ou privés. 


\title{
Force localized interaction sensing system for HYDRÖ̈D humanoid robot
}

\author{
M. Tahoun, A. Tayba, S. Alfayad, A. Wahdan and L. Chassagne, Member IEEE
}

\begin{abstract}
In this paper, we present a new type of low cost force localized interaction sensor dedicated to robot interactive sensing system. The design of the sensor is based on mechanical deformation and mathematical models. It includes the capability to be integrated easily in robotic limbs. This sensing system is based on the coupled results obtained from six well-located force sensors in order to measure the magnitude, position and orientation of the interacting force. The mathematical model to calculate the force from the measurements is fully detailed. A prototype in the 2Dimensional plan is built. An experimental proof of concept setup is illustrated and sets of measurements illustrate force sensing results. A commercial sensor based on another technology is used for reference. The comparison with the reference shows very close results on a tested range up to 50 $\mathrm{N}$. The standard deviation of the difference between both sensors is lower than $1 \mathrm{~N}$ on a tested range of $50 \mathrm{~N}$ and for several angles of the applied force, demonstrating the relevance of the new sensor.
\end{abstract}

\section{Index Terms — Force Sensors, Humanoid Robots,}

\section{INTRODUCTION}

$\mathrm{H}$ umanoid robot researches have rapidly evolved over the last decades. Therefore, many projects have been launched to develop new humanoids aimed to interact with human environment. Providing services such as disabled person carrying, housework assistance are classical examples for smooth interactions. Moreover, some projects aim more complex tasks such as ultimate helpers in man-made or natural disasters [1-4]. In this context, safe robot motion control is the point that slows down the design of the humanoid robot, and the fulfillment of these kinds of tasks. However, it is unrealistic to limit these technologies behind the walls of

This work was sustained by iCode, the Institut pour le Contrôle et la Décision de l'Université Paris-Saclay.

Samer Alfayad, Ahmad Tayba and Luc Chassagne are with the LISV laboratory of the University of Versailles Saint-Quentin, 10-12 avenue de l'Europe, 78140 Velizy, France (corresponding authors phone: +33 1392549 84; e-mail: \{ luc.chassagne\}@ uvsq.fr).

Abdelmoneim Wahdan is from AASTMT, the Arab Academy for Science, Technology \& Maritime Transport, Cairo, Egypt.

Mohamed Tahoun is an internship student depending on both LISV/UVSQ and AASTMT. research or limit its application just to do a trivial task. It is very relevant for the humanoid robots to control interactions between humanoids and their surrounding environments. That can be the robot itself, human operator or any static or dynamic obstacles [5-7]. These smooth behaviors require high performance levels for a safety related control systems. The parameters about position and velocity, trajectory, collision avoidance, safety-related autonomous decisions, are to be fully controlled for stability and safety.

In order to give robots a role in the external world as well as dealing with the human life, the safety in pHRI (physical Human-Robot Interaction) must be ensured. Nowadays, safe interaction is still an open topic of research. At the same time, various interaction control theories and motion generation strategies have been developed with the intention to realize safe robot motion control [8]. The researches have been focused to overcome this issue, especially on human-robot collision. The impact of the forces occurred due to the humanrobot collision can be diminished through designing a lightweight robots [9], compliance control (complaint actuation) [10-11] or impedance control [12]. To apply any of these or other solutions, it is needed to know about the force resulting from the collision. All of predictable and unpredictable interactions have to be sensed and identified by the robot. Accordingly, safe interaction cannot be achieved without perception of the interaction localization, the type of interaction and the magnitude of the resulting force from interaction. With this in mind, human-safe robots should have a tactile sensing system.

This paper presents a new concept to tackle the problem of pHRI for the home-made new humanoid robot called HYDROïD [13-14], through developing a force localized interaction measurement system. HYDROiD is an underdevelopment home-made humanoid robot of our laboratory. It has been designed with more than 40 degrees of freedom totally actuated by the hydraulic pressure except for the head. This robot has $1.85 \mathrm{~m}$ of height, weighs $120 \mathrm{~kg}$ and is designed to walk with a maximum speed of $1.2 \mathrm{~m} / \mathrm{s}$. Hence, the collision avoidance becomes greatly important especially because some uses of the robot are dedicated to help disabled person. In addition, this new generation of humanoid robot, which is associated with very high torque, increases the need to a safe interaction mainly in unstructured human environment.

A lot of transducers have been used in basic tactile sensing [15]. Generally speaking, most of these systems are targeting the end-effectors for gripping and manipulating rather than covering the whole body. However, the contact position of the robot with the external world is needed to be monitored to fulfill the minimum requirements of pHRI. 
In the last two decades, numerous experiments on the development of the robot skin have been done to overcome safe interaction concerns. O'Neill et al. demonstrated a stretchable smart skin sensor based on three layers of flexible and stretchable materials [16]. The layers are made of Agnylon: silver-coated conductive fabric, non-conductive perforated cloth and $1.75 \mathrm{~mm}$ polydimethylsiloxane (PDMS) substrate with a uniform, bonded carbon nanotube (CNT)PDMS coating. The layers are pasted together by nonconductive PDMS glue. Four conductive nodes were fixed to the corners of the skin. The wire of each node and the top layer were connected directly to the pins of the microcontroller. The drawback of this design is the need to be calibrated after fixing and stretching.

Minato et al. have designed their skin for the CB2 child robot by integrating small thin Polyvinylidene fluoride (PVDF) films beneath the skin [17]. The sensors are placed over the robot body which lead to non-molecularity of the system. Then, the tactile sensors are put between the outer silicon cover and the urethane foam that cover the mechanical parts. Due to the rate of deformation of the cover, the output of the sensors is changed providing the tactile information. The entire body of the robot is covered by 197 tactile sensors which is a big number for covering a baby sized robot.

Maria et al. demonstrated a distributed 6 axis F/T tactile sensing based on optical sensors [18]. The skin is structured of a matrix of opto-electronics components covered by black deformable silicon layer. The photo-diode always illuminates to bottom face of the elastic layer. The intensity of the reflected signals to the optic devices is changed according to the deformation of the elastic layer when an external force is applied. The force got from this structure is up $3.5 \mathrm{~N}$, and the torque is up to $10 \mathrm{~N} . \mathrm{mm}$ which is a low range for a humanoid robot. Others optical sensors have been also developed with fiber optics technology, for example the one exposed by Arnaldo et al. [19] used in a prototype of rehabilitation exercise of the leg. It uses the coupling principle of fiber optics by detecting the amplitude variation of light is a misalignment appears. The advantage of strain jauge fibered technology is that it has been extensively studied for plenty of others fields like survey in buildings for example and then durability has been explored which can be a important criteria for long term embedded systems [20].

Iwata et al. implemented tactile interface to be applied to the WENDY humanoid robot [21]. The robot body is shielded by inflexible covers that is roofed by Force Sensing Resistor (FSR) touch sensors. A six-axis force torque sensor is linked to the cover that is lying on the robot body on one single point. Through this design, accurate contact position and resulting forces and torques are measured with high accuracy. According to shield the cover with the FSR, the noise level and hysteresis are high.

Maggiali et al. presented artificial skin for cover the whole body of a humanoid robot [22-23]. In this design, capacitive pressure transducers have been implemented using capacitance to digital converter integrated circuits (CDC). This system is based on a mesh network of sensors having triangle shape. Each triangle has a doubled layer flexible PCB with 3 $\mathrm{I} / \mathrm{O}$ ports and 12 circular pad that is covered by a conductive ground plane of silicon rubber. Each mesh network can be structured with up to 16 triangles connected to the same micro-controller. The range of measured forces is small. In addition, the sensitivity might be reduced according to the fixation of the silicon rubber layer, and the system is affected by temperature.

Mittendorfer and Cheng propose a modular multi-modal tactile sensing module named HEX-O-SKIN [24]. This hexagon module is interconnected together to cover large areas of robotic structures. Each module is composed of hexagon rigid PCB with four photo-transistors, a three-axis accelerometer and seven temperature sensors. Accordingly, the so-called HEX-O-SKIN is capable of sense pre-touch, light touch and temperature. The data processing is done completely on each module whereas the HEX-O-SKIN modules interconnected together using communication channels to the adjacent modules. This system is impractical during rough physical interaction because a failure to interconnections between the modules is expected.

In this paper we propose a new solution based on six welllocated force sensors and a mathematical model. The paper is organized as follows. Section II presents the basic specifications needed for the skin, the system design, and the mathematical model. The section III describes the first prototype. Section IV shows the configuration of the test platform and experimental results that presents the first prototype experiments. The conclusion and future work is ultimately discussed in Section V.

\section{SYSTEM DESIGN}

\section{A. Skin specifications}

We give firstly some specifications about the tactile sensing systems we are targeting. First, the system should be able to identify agile and massive interactions. The sensitivity must be as low as $0.1 \mathrm{~N}$ and the range up to $50 \mathrm{~N}$. Second, the developed system should have a compact and comfortable design, in order to be relevant for any arbitrary surfaces. It must be able to fit into the robot shell design. In addition, because the noise and hysteresis depend heavily on the complexity of wiring, our system is needed to be as simple as possible in its wiring network. We aim also to minimize the power consumption of the sensor, because it is important to decrease the power consumption of the whole robot that can contains many tactile sensing elements. Moreover, as our system will be used for safe interaction and collision avoidance in human environment, it should obviously have fast response time to help the robot controller system to take a fast decision. Finally, all the required specifications mentioned above must be implemented in a low cost system, enabling us to cover the entire body of the robot with minimum price and allows a large robotic population to use this system.

\section{B. Architecture of the system}

The principle of the measurement is based on the curved beam principle as presented in Fig. 1. Triangles 1 and 2 (respectively red and green) represent three axis force sensors. They are fixed to a curved beam, a simple mathematical model that generates the magnitude of the force acting on the surface 
as well as its application point and orientation. The force $F$ in Fig. 1 is considered as an arbitrary interaction force between the robot and the external environment producing a little displacement for the curved rigid beam. As a result of this displacement, the projected forces $\mathrm{F}_{\mathrm{Y} 1}, \mathrm{~F}_{\mathrm{Z} 1}, \mathrm{~F}_{\mathrm{Y} 2}$ and $\mathrm{F}_{\mathrm{Z} 2}$ will be measured based purely on the deformation of the external body of the two sensors. $l$ and $a$ in Fig. 1 refer respectively to the distance between the 2 force sensors and the distance between the sensor 1 and the force point of application.

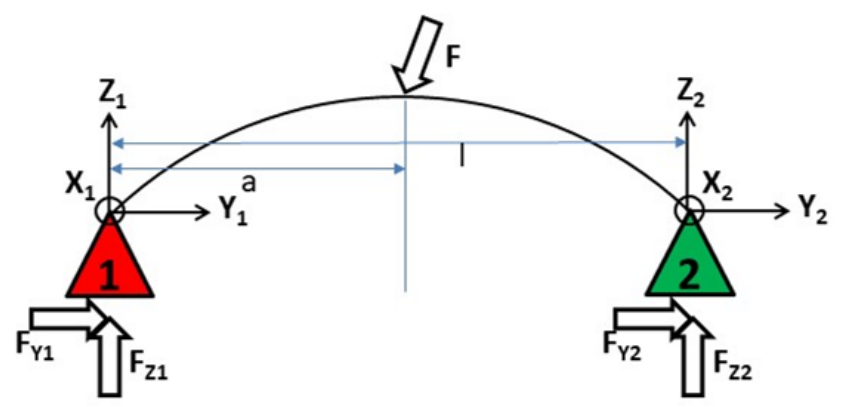

Fig. 1. Sketch about the curved beam standing on two vertical optoforce sensors. 1 is the distance between the two sensors, and a is the projection along $\mathrm{Y}$ axis of the distance between sensor 1 and the applied force.

As our reference point will be at the center $\mathrm{O}$ of the circular surface, therefore, we have changed the positioning angle of the two sensors, to have the direction of the force $\mathrm{F}_{\mathrm{Z} 1}$ and $\mathrm{F}_{\mathrm{Z} 2}$ normal to the outside circular surface of the system. In this case, the angle $\alpha$ becomes $60^{\circ}$ and the two forces $\mathrm{F}_{\mathrm{Z} 1}$ and $\mathrm{F}_{\mathrm{Z} 2}$ come across at the center $\mathrm{O}$ of the system as shown in Fig. 2.

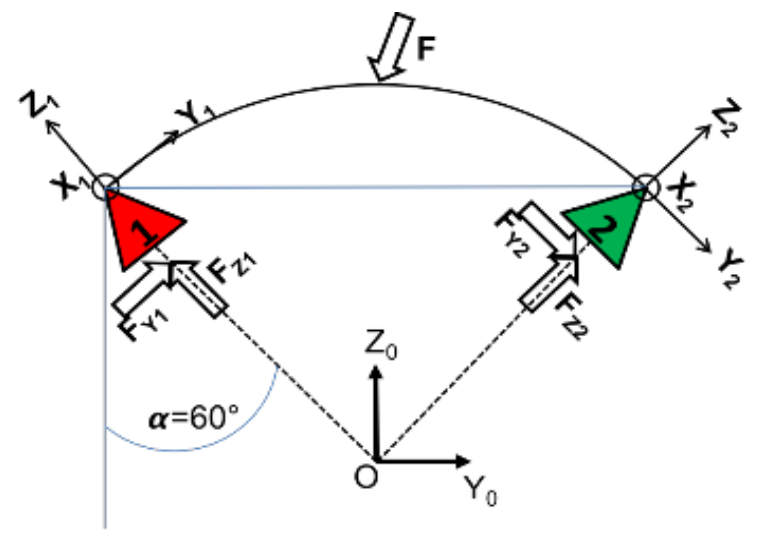

Fig. 2. Sketch about the curved beam standing in the hypothesis of two inclined Optoforce sensors.

In order to cover all the circumference of the robot limb which is always considered as a cylindrical or conical shape shell, a set of $n$ 3D force sensors are distributed as shown in Fig. 3. In this example a three sensors set has been used just for explanation purposes and to have a clear figure.

Our system is composed of an inner fixed part, called stator, used to fix the $n$ force sensors. On the Fig. 3, it is represented by the smaller black circle. It is the connection part between the robot limb and the system. This stator will be replaced directly by the robot mechanical parts in order to reduce as much as possible the size of our system. The outer circular shape of the system, represented by the larger black circle, is a mobile part that is directly exposed to any external interaction force $F$. This external force causes a little displacement to the rigid outer part of the skin system in the same direction than the force. The red dotted circle in Fig. 3 represents this displacement.

$R$ is the radius of the external circular part, $Y_{F}$ and $Z_{F}$ are the location of the force relative to the frame $\left(Y_{0}, Z_{0}\right) . \alpha_{1}, \alpha_{2}, \alpha_{3}$ are the angles between the external force and the $Z$ axis of each sensor. $\alpha_{0}$ is always equal to $\alpha_{1}$.

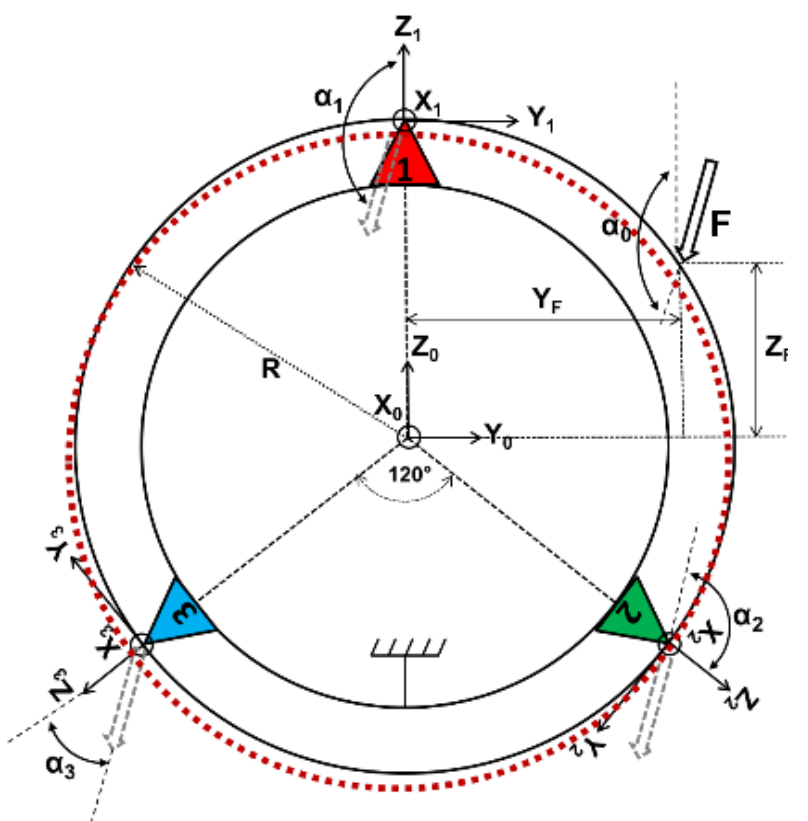

Fig. 3. Sketch of 3D OptoSkin design architecture with an example with three force sensors.

\section{Mechanical model}

To generalize our model approach, a 3D cylindrical shape have been used, as shown in Fig. 4, representing the interaction detection system covering the robot limb. This outside shape can be modeled also as a conical shape. As it can be seen in Fig. 4, the outer cylinder is standing on $n$ force sensors (blue triangles) equally distributed from each side. $i$ and $j$ represents the number of sensor on left and right side respectively. These sensors are placed in a way that the $\mathrm{Z}$-axis of the sensors intersects the origin center of each side. $\alpha_{i}$ and $\alpha_{j}$ represent the angle between Z-axis of the system and sensor $i$, and $j$ respectively. The interaction force is represented by $F$ with its point of application $P\left(X_{F}, Y_{F}, Z_{F}\right)$. The length $L$ represents the length of the covered limb and $R$ represents the radius of each side of the cylinder. 


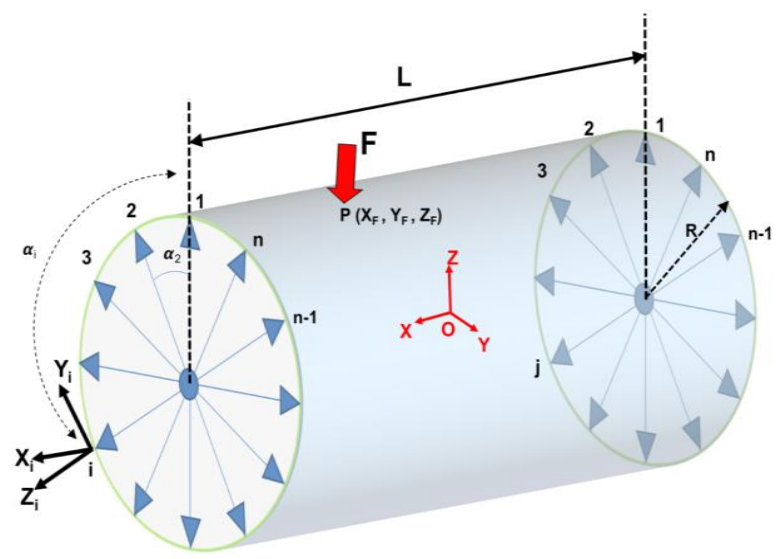

Fig. 4. OptoSkin general mechanical model.

Based on the third Newton law, the magnitude of the interaction force can be calculated as follow:

$$
\begin{aligned}
& F_{x}+\sum_{i=1}^{n} F_{x i}+\sum_{j=1}^{n} F_{x j}=0, \\
& F_{y}+\sum_{i=1}^{n} F_{y i} \cdot \sin \left(\alpha_{i}\right)+\sum_{j=1}^{n} F_{y j} \cdot \sin \left(\alpha_{j}\right)=0, \\
& F_{z}+\sum_{i=1}^{n} F_{z i} \cdot \cos \left(\alpha_{i}\right)+\sum_{j=1}^{n} F_{z j} \cdot \cos \left(\alpha_{j}\right)=0 .
\end{aligned}
$$

Using the equations 1, 2, and 3, the direction of the interaction force can be obtained as follow:

$$
\begin{aligned}
& \alpha_{F}=\arctan \left(\frac{F_{y}}{F_{z}}\right), \\
& \theta_{F}=\arctan \left(\frac{F_{x}}{\sqrt{F_{y}^{2}+F_{Z}^{2}}}\right) .
\end{aligned}
$$

The position $X_{F}, Y_{F}$ and $Z_{F}$ of the interaction force $F$ can be obtained by using the moment equilibrium law around the center O:

$\sum M_{x}=0$ gives:

$$
\begin{aligned}
& F_{y} \cdot Z_{F}+F_{z} \cdot Z_{F} \\
& \quad+R \cdot \sum_{i=1}^{n}\left(F_{y i}+F_{z i}\right) \cdot \sin \left(\alpha_{i}\right) \cdot \cos \left(\alpha_{i}\right) \\
& \quad+R \cdot \sum_{j=1}^{n}\left(F_{y j}+F_{z j}\right) \cdot \sin \left(\alpha_{j}\right) \cdot \cos \left(\alpha_{j}\right)=0,
\end{aligned}
$$

$\sum M_{y}=0$ gives:

$$
\begin{aligned}
& F_{x} \cdot Z_{F}+F_{z} \cdot Z_{F}+R \cdot\left(\sum_{i=1}^{n} F_{x i} \cdot \cos \left(\alpha_{i}\right)+\sum_{j=1}^{n} F_{j i} \cdot \cos \left(\alpha_{j}\right)\right) \\
& +\frac{L}{2}\left(\sum_{i=1}^{n} F_{z i} \cdot \cos \left(\alpha_{i}\right)+\sum_{j=1}^{n} F_{z i} \cdot \cos \left(\alpha_{j}\right)\right)=0 \\
& \sum M_{z}=0 \text { gives }
\end{aligned}
$$

$$
\begin{aligned}
& F_{x} \cdot Y_{F}+F_{z} \cdot X_{F}+R \cdot\left(\sum_{i=1}^{n} F_{x i} \cdot \cos \left(\alpha_{i}\right)+\sum_{j=1}^{n} F_{j i} \cdot \cos \left(\alpha_{j}\right)\right) \\
& +\frac{L}{2}\left(\sum_{i=1}^{n} F_{y i} \cdot \cos \left(\alpha_{i}\right)+\sum_{j=1}^{n} F_{y i} \cdot \cos \left(\alpha_{j}\right)\right)=0
\end{aligned} .
$$

\section{FIRST PROTOTYPE DESCRIPTION}

A first experimental prototype has been developed. It contains three force sensors. The mobile part (the stator as explained above) is designed in two symmetrical parts to cover the three sensors without destroying the external surface of the sensor. After assembly, these two parts have been fixed together to form a single fixed part. The inner part which carries the three sensors is designed as a hollow cylinder, where a small shaft is representing the arm of the robot, that will be integrated in the future. All these parts are designed with CATIA software and manufactured from a customized plastic using the $3 \mathrm{D}$ printing technology. A CAD model for our prototype is shown in Fig. 5 top hand side and the plastic first prototype in Fig. 5 middle hand-side.
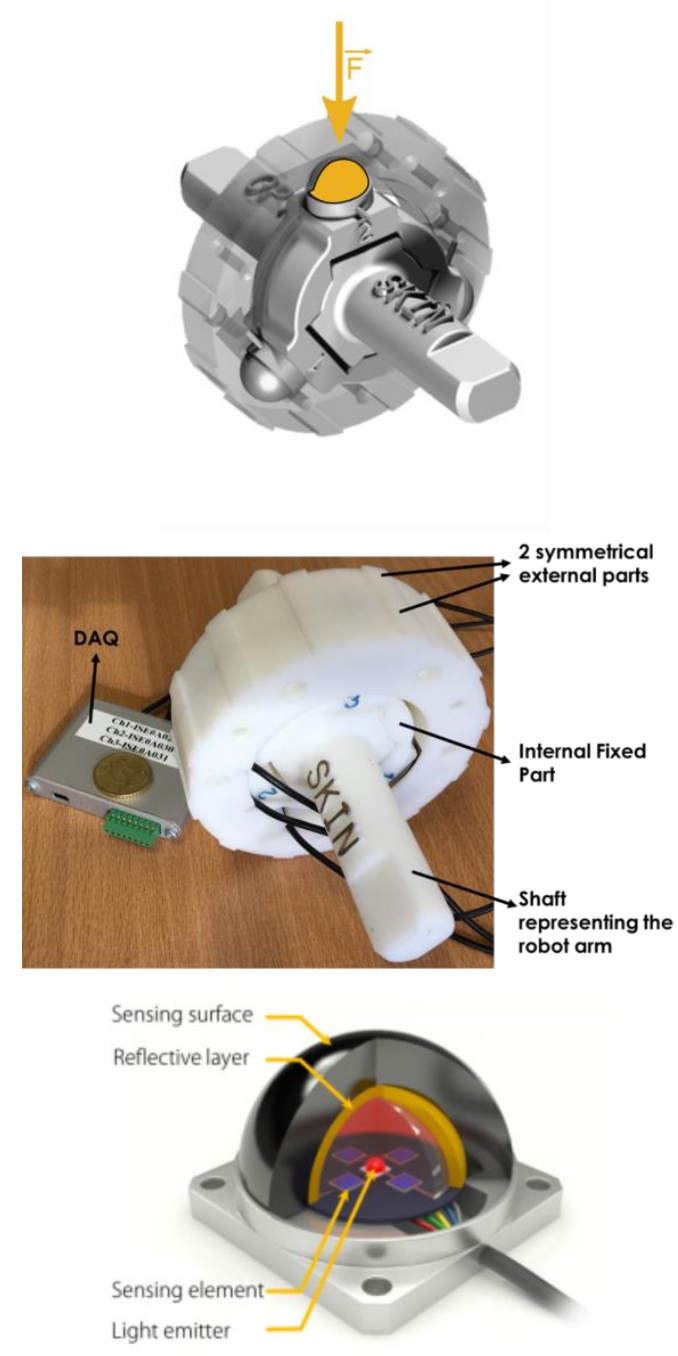

Fig. 5. Top: CAD of OptoSkin first prototype; Center: First prototype made with 3D printing technology; Bottom: Optoforce sensor [25] 


\section{EXPERIMENTAL RESULTS}

As illustrated above, the measuring principle in our system is based on a three axis force sensor. There are many types and models of force sensors and we have chosen a three axis optical force sensor, illustrated Fig. 5 bottom, called OptoForce [25]. An infrared light is used to identify any deformation occurred to the outer surface of the sensor. In addition, these sensors are characterized by a high degree of elasticity and durability due to the isolation between the deforming surface and the sensing elements. This sensor have a $2.5 \mathrm{mN}$ resolution and can sense up to $40 \mathrm{~N}$ with a maximum single axis overload up to $200 \%$. The dimensions of this sensor are $17 \mathrm{~mm} \times 25 \mathrm{~mm} \times 25 \mathrm{~mm}$ with a weight of $23 \mathrm{~g}$.

Fig. 6. Optoforce sensor [23].

The OptoForce DAQ (data acquisition card) has a compact size which facilitate its integration in the robot body. Moreover, four sensors can be connected to the same DAQ, which simplify the wiring problem as mentioned in the specifications section. This DAQ can be interfaced with the computer as shown in the Fig. 7. All the reading and filters of the forces are made into the DAQ. Furthermore, we have developed our own software that interfaces with the DAQ via USB. The software is composed of many modules to read the force data and integrate the sensors data into our mechanical model. Finally, the module displays the sensor data, and its parameters like system response and resultant force in a real time graph. Everything can be saved and exported. This software has been developed through Qt cross platform software using $\mathrm{C}++$. The maximum sampling frequency between the DAQ and the PC is $1 \mathrm{kHz}$ which leads to very comfortable response time for our our skin system. Finally, Fig. 6 bottom shows a screenshot of the developed application for the OptoSkin.
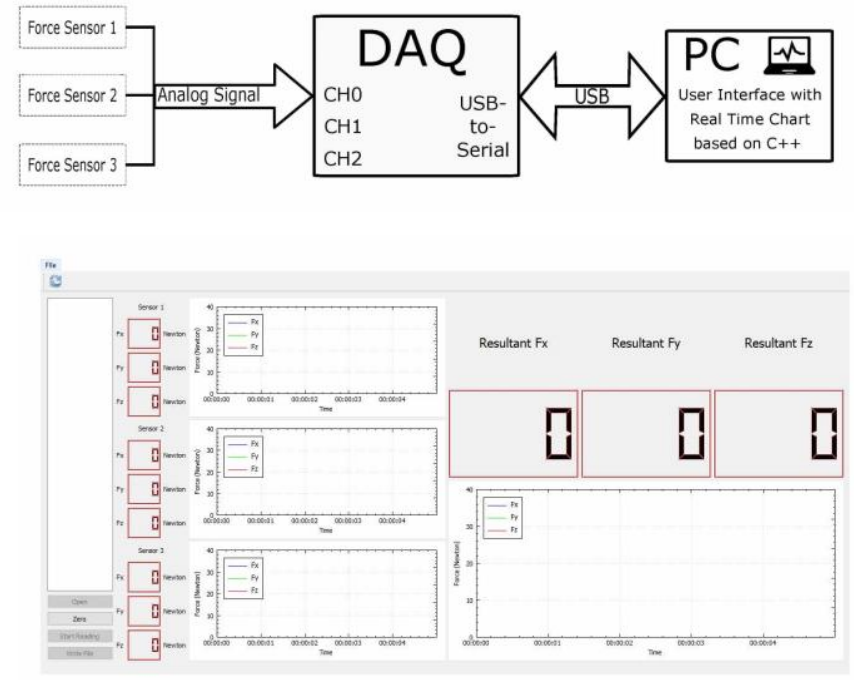

Fig. 6. Top: OptoSkin control block diagram; Bottom: Screenshot for GUI of the developed software

\section{A. Experimental setup}

The first prototype shown in Fig. 5 is integrated in the experimental setup for force testing illustrated in Fig. 7. The setup is composed of a milling machine, a force sensor and plastic support parts to be the stands of the force sensors, illustrated on the bottom part. These stands allow us to interact with the system with different angles (90 and 45 degrees). At the beginning of the experiment, the prototype is fixed on the table of the milling machine and locked on the shaft that represents the robot arm. Then, the force sensor tester is mounted with the stand chosen based on the required angle of interaction. The stand is installed and locked upside down in the drill chuck. Finally, the force is applied to the system perpendicularly using the vertical feed hand-wheel towards the table.

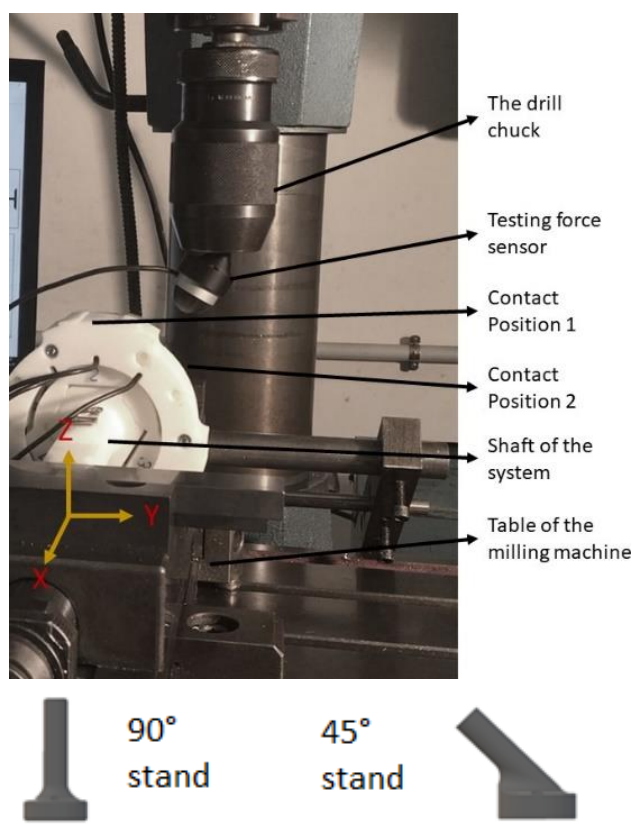

Fig. 7 Top: setup of the experiment with the milling machine; Bottom: stands of the force sensor tester

The force sensor used for testing and validating our system is an Optoforce 3-axis force sensor (called Tester in the following) that is interfaced separately to its original dedicated software. Actually, in the following experiments we are concerning principally about the resultant force of the Z-axis of our system. Note that the characteristics of the Optoforce sensor are a range of temperature between $-40^{\circ} \mathrm{C}$ to $+80^{\circ} \mathrm{C}$ and a non-linearity below $5 \%$. The temperature sensitivity is not declined in the datasheet.

All the measurements (Optoskin and the Tester) are done at an updating frequency of $100 \mathrm{~Hz}$. The data from both the Optoskin and the Tester are synchronized. The applied pressure is obtained as the force measured by the Tester. The resultant force is obtained by substituting the output force for each sensor integrated in the Optoskin system using the mechanical model developed in the previous section. 
The experiments are performed in two different configurations with several trails for each one. In the first configuration, the pressure is applied perpendicularly to the Optoskin system exactly over the sensor 2 in the system. In the Fig. 7, it is the contact position 1 . In the second configuration, the pressure is applied diagonally by using $45^{\circ}$ stand of the Tester on the Optoskin between sensor 2 and sensor 3 , which is noted contact position 2 as shown in Fig. 7.

\section{B. Results with force perpendicularly applied}

In a first step, several forces are developed to compare both sensors Optoskin and Tester. A set of step forces, respectively $15 \mathrm{~N}, 20 \mathrm{~N}, 30 \mathrm{~N}, 40 \mathrm{~N}$ and $50 \mathrm{~N}$, is applied to the contact point 1. Fig. 8a shows the results of the comparison between both sensors. Fig. $8 \mathrm{~b}$ plot the difference between both sensing systems.

a)

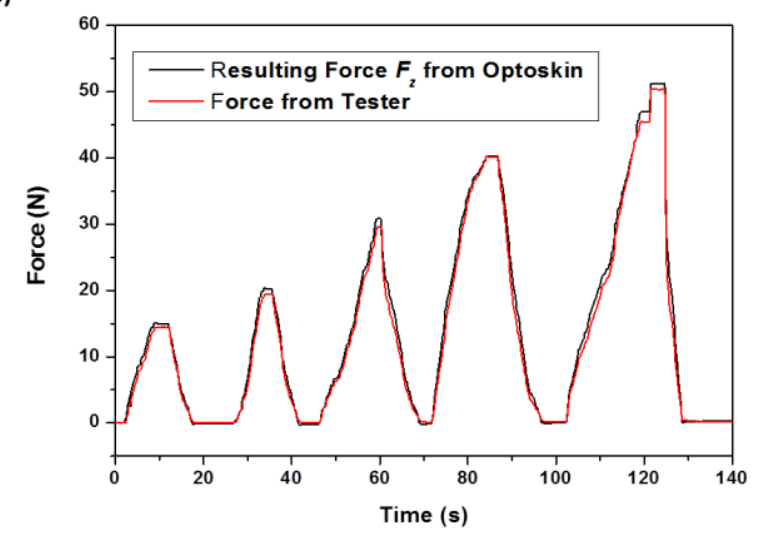

b)

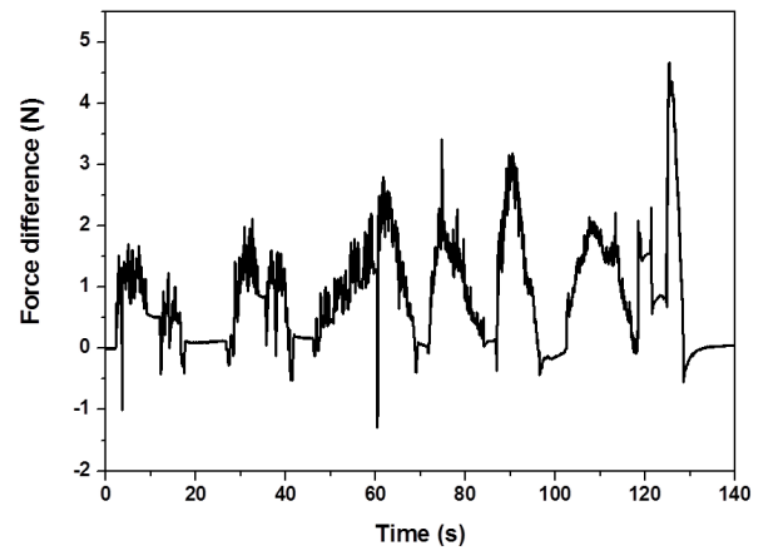

c)

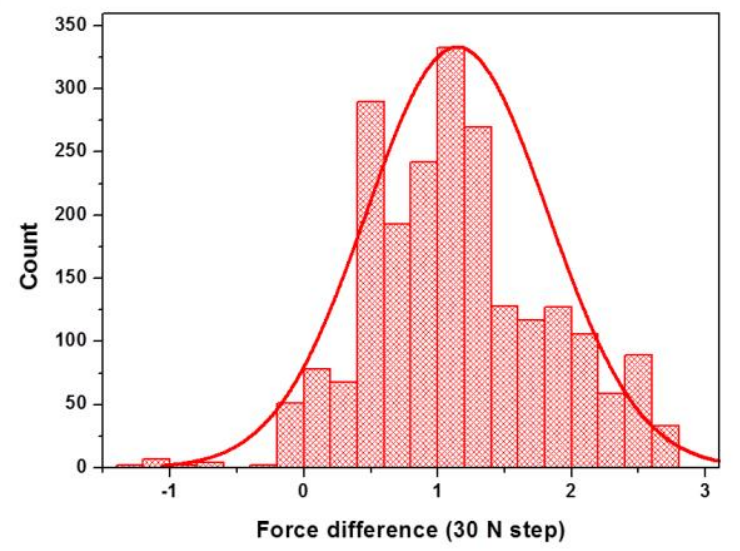

Fig. 8. a) Comparison between the two sensors for applied forces of $15 \mathrm{~N}, 20$ $\mathrm{N}, 30 \mathrm{~N}, 40 \mathrm{~N}$ and $50 \mathrm{~N}$; b) Difference between both sensors; c) Histogram of the force difference for the $30 \mathrm{~N}$ step.

We can see that both results are very close. The resultant force is almost the same as the pressured force with minimum and maximum absolute error respectively $-1.2 \mathrm{~N}$ and $+4.6 \mathrm{~N}$, mainly in transitory areas. The absolute error is different according to the range of the applied pressure. The mean value of the force difference is $0.87 \mathrm{~N}$ on all over the experiment. To be finer in the comparison, the histogram of the difference has been plot for one step, the $30 \mathrm{~N}$ step, illustrated in Fig. 8c. We can see that the distribution of the difference between both sensors is quite Gaussian shape. The standard deviation is estimated to $0.68 \mathrm{~N}$. This standard deviation can be considered as the quadratic sum of the noise of both sensors because it is hard to separate the influence of the two systems. We consider that it is the combined resolution, but it means that it is a high value for the intrinsic resolution of our sensor.

In order to verify that the system can reproduce its performances, we launch a second set of experiments. The same setup is used. A force of $10 \mathrm{~N}$ is gradually applied on the system and then released also gradually. Five trials are launched and recorded. The Fig. 9a represents the trial 1 and Fig. $9 b$ the force difference for the five trials. The timing of the pressure excitation is quite hard to control accurately. The curves can presents pattern difference, especially to the releasing part. Nevertheless, we can see that the sensor is quite repeatable in the results for the same process of excitation, with mean value and standard deviation of the difference in the same order of magnitude, less than $1 \mathrm{~N}$. The mean value of the difference can be an absolute reference bias because both sensors are not absolute calibrated sensors. Furthermore, the applied source is not fully repeatable at Newton scale and can also induce some discrepancy on the results. Finally, the impact of the temperature is hard to estimate for this proof of concept. The reference OptoForce has a temperature using range between $-40^{\circ} \mathrm{C}$ and $+80^{\circ} \mathrm{C}$ but the influence of the temperature is unknown. For the Optoskin, the mathematical model includes measurements from several points of measures that can depend on temperature or other environment parameters but it is quite impossible to estimate the 
uncertainty of each point. Nevertheless in the case of the aimed application for detection of interaction with human or systems, the true value of the force is not the objective, a difference of few Newton is then acceptable.

a)

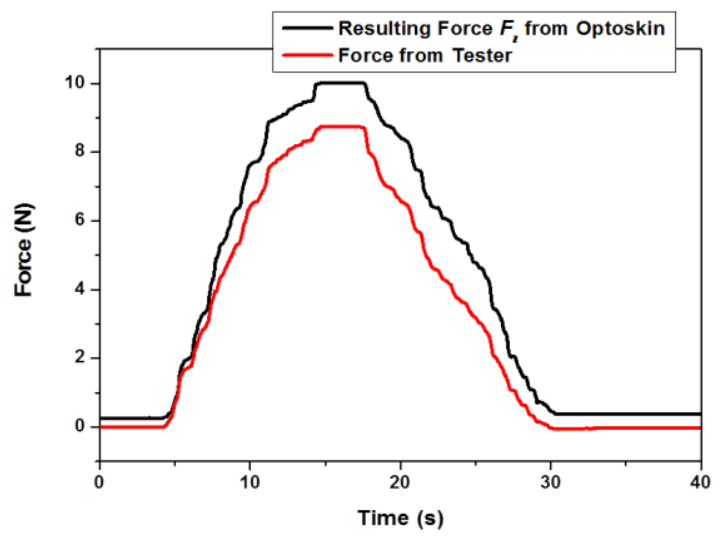

b)

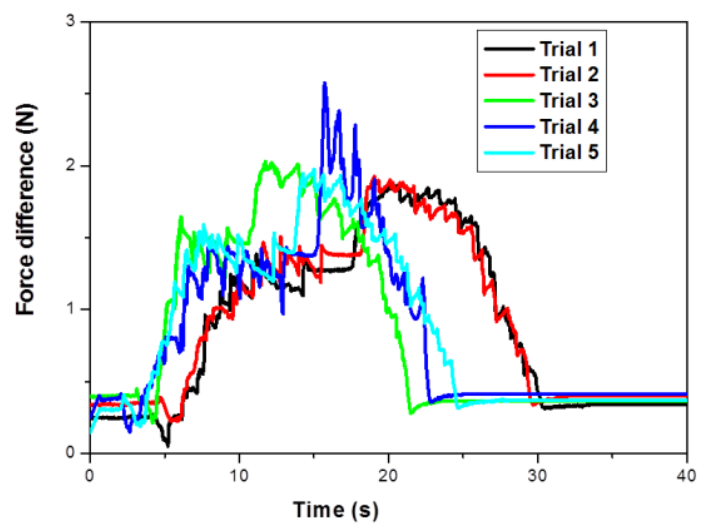

Fig. 9. a) Comparison between the two sensors for an applied force of $10 \mathrm{~N}$ for the first trial; b) Difference between both sensors for five consecutive trials.

\section{Results with force diagonally applied}

In this second configuration the pressure was applied diagonally by using $45^{\circ}$ stand of the tester on the OptoSkin between sensor 2 and sensor 3 (contact position 2) as shown in Fig. 7. The applied force was calculated by making a projection of the $Z$ axis force on the $Z$ axis of the hole system. Similarly, the resultant force on the $Y$ axis was calculated by making a projection of the $Y$ axis force of the whole system. The Fig. 10, parts a, b, c, d represents respectively the comparison between both sensors on $Y$ axis, the difference of the two measurements, and the comparison and the difference on the $Z$ axis. a)

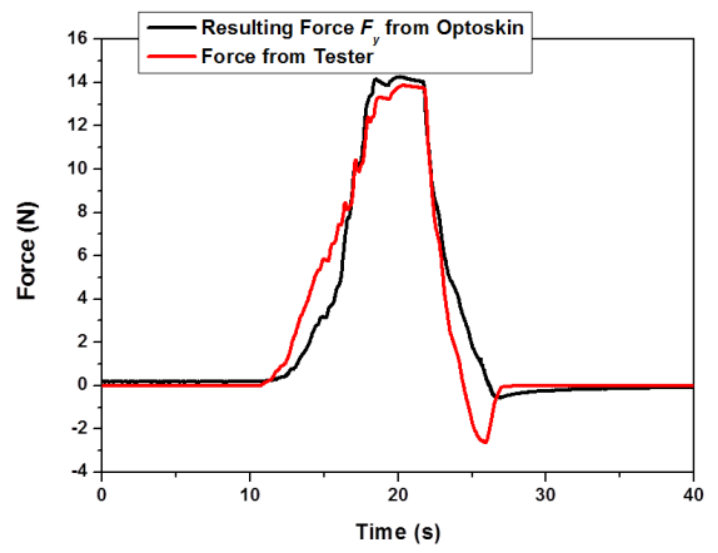

b)

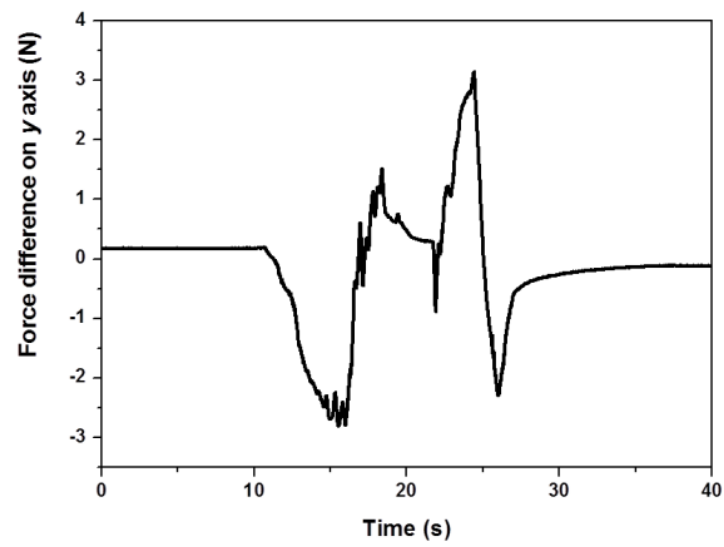

c)

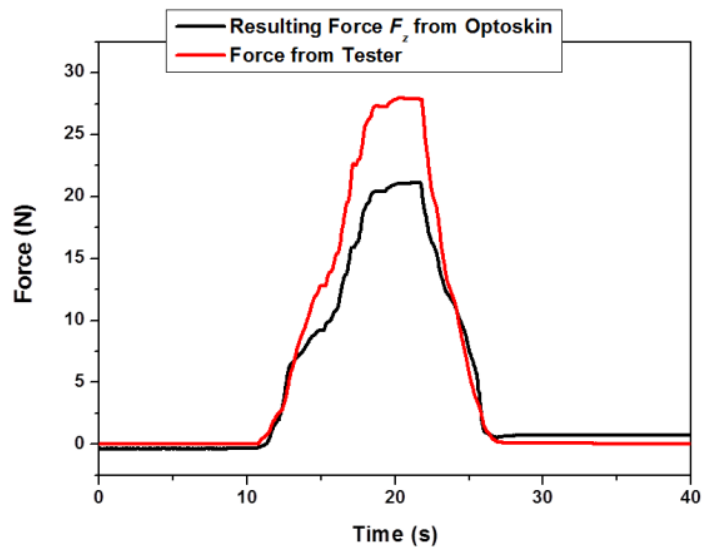


d)

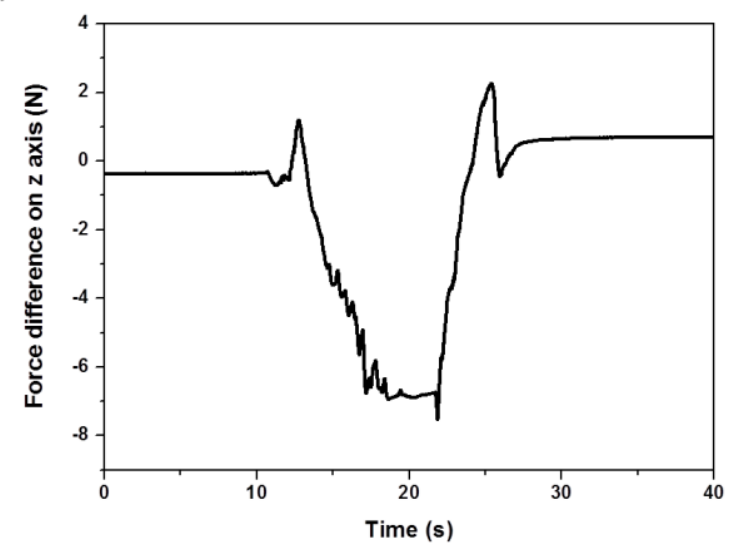

Fig.10. a) Comparison between the two on Y axis; b) Difference between both sensors for $Y$ axis; c); Comparison between the two on $Z$ axis; d) Difference between both sensors for $Z$ axis

As previously, we can see that the sensor is reacting correctly in this diagonally configuration. Nevertheless, the results present an observable difference for the $F_{z}$ estimation that is higher than previous results. We estimate that this is an effect related to a little slipping between the inner part and the outer part. It induces that the 3D model is not fitted perfectly. We plan to add some springs between the inner and the outer part in the future design to avoid this slipping.

\section{CONCLUSION AND PERSPECTIVES}

In this paper, we present a new sensing system composed of modular force localized sensors used for powerful humanoid robots. The first prototype of the system shows the reliability with an acceptable range of error, compared to a reference sensor. The analytic and robust mathematical model has been developed, to obtain the real interacting forces. Moreover, the force sensors, used in creating our system, were chosen to cover a high range of forces with high resolution.

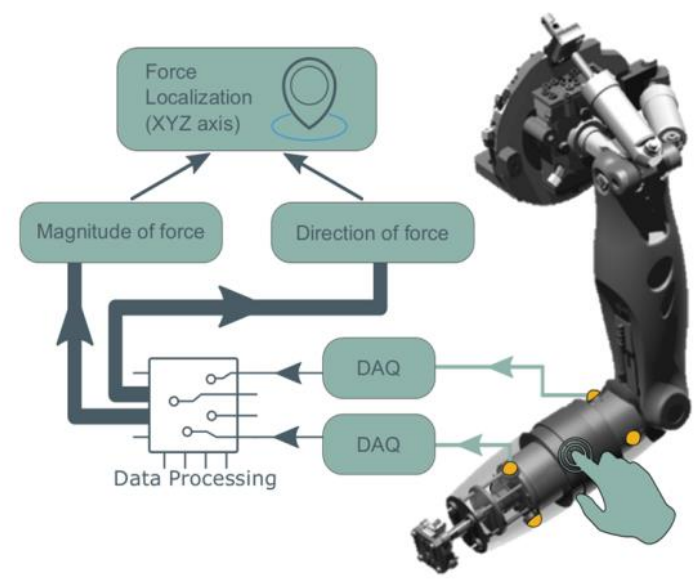

Fig.11. Future design of the full interaction for HYDROïD seven degrees of freedom arm.
The experimental results show good performances compared to a commercial reference sensor. Step forces as high as $50 \mathrm{~N}$ have been measured with repeatability demonstration and standard deviation lower than $1 \mathrm{~N}$.

We plan to improve the fixation of the sensors to reduce the friction between the sensors and the output cover. It should lead to minimize the absolute error. Furthermore, an extensive study of the influence parameters (temperature, uncertainties of each measurement points) will developed to estimate the accuracy of the final prototype. As well as, we will expand our system to cover the area of the robot lower arm. The proposed design for the expanded system for HYDRÖ̈D's seven degree of freedom (DOF) arm is shown in Fig. 11. Finally, the export data of the system will be used as an input for the robot controller to ensure the essential principle that is safe interaction.

\section{REFERENCES}

[1] Kaneko K, Kanehiro F, Morisawa M, Akachi K, Miyamori G, Hayashi A and Kanehira N, Humanoid robot HRP-4-Humanoid robotics platform with lightweight and slim body, IEEE International Conference on Intelligent Robots and Systems. pp. 4400-4407, 2011.

[2] Sakagami Y, Watanabe R, Aoyama C, Matsunaga S, Higaki N and Fujimura K, The intelligent ASIMO: system overview and integration, IEEE/RSJ International Conference on Intelligent Robots and Systems 3, 2011.

[3] Ogura Y, Aikawa H, Shimomura K, Morishima A, Lim HO and Takanishi A, Development of a new humanoid robot wabian-2 : 76-81, 2006.

[4] Nishiwaki K, Kuffner J, Kagami S, Inaba M and Inoue H (2007) The experimental humanoid robot $\mathrm{H} 7$ : a research platform for autonomous behaviour. Philosophical transactions. Series A, Mathematical, physical, and engineering sciences 365: 79 - 107, 2007.

[5] Haddadin S, Towards safe robots, volume 90. Springer, 2014.

[6] Luca AD and Flacco F, Integrated control for phri: Collision avoidance, detection, reaction and collaboration. In: 2012 4th IEEE RAS EMBS International Conference on Biomedical Robotics and Biomechatronics (BioRob). pp. 288-295, 2012.

[7] Haddadin S, Albu-Schäffer A and Hirzinger G, Requirements for safe robots: Measurements, analysis and new insights. The International Journal of Robotics Research 28(11-12): 1507 - 1527, 2009.

[8] Haddadin S, Towards safe robots, Approching Asimov's 1st Law, volume 90. Springer, 2014.

[9] Hirzinger G, Sporer N, Albu-Schaffer A, Hahnle M, Krenn R, Pascucci A and Schedl M, Dlr's torque-controlled light weight robot iii-are we reaching the technological limits now? Proceedings of International Conference on Robotics and Automation (Cat. No.02CH37292), volume 2. pp. 1710 - 1716 vol.2. DOI:10.1109/ROBOT.2002.1014788, 2002.

[10] Lim B, Lee M, Kim J, Lee J, Park J, Seo K and Roh K, Control design to achieve dynamic walking on a bipedal robot with compliance. In: 2012 IEEE International Conference on Robotics and Automation. pp. 79-84. DOI:10.1109/ICRA.2012.6224882, 2012.

[11] Zinn M, Khatib O, Roth B and Salisbury JK, Playing it safe [humanfriendly robots]. IEEE Robotics Automation Magazine 11(2): 12-21, 2004.

[12] Fasse ED and Broenink J, A spatial impedance controller for robotic manipulation. IEEE Transactions on Robotics and Automation 13(4): 546556. DOI:10.1109/70.611315, 1997.

[13] Alfayad S, Ouezdou FB and Namoun F, New three DOF ankle mechanism for humanoid robotic application: Modeling, design and realization, Journal of Mechanical Design : 4969 - 4976, 2009.

[14] Alfayad S, Ouezdou FB, Namoun F, Bruneau O and Hénaff P, Three DOF hybrid mechanism for humanoid robotic application: Modeling, design and realization, IEEE/RSJ International Conference on Intelligent Robots and Systems, IROS 2009 : 4955 - 4961, 2009. 
[15] Lee $\mathrm{M}$ and Nicholls $\mathrm{H}$, Review article tactile sensing for mechatronics a state of the art survey, Mechatronics 9(1): $1-31$. DOI:https://doi.org/10.1016/S0957-4158(98) 00045-2, 1999.

[16] O’Neill J, Lu J, Dockter R and Kowalewski T, Practical, stretchable smart skin sensors for contact-aware robots in safe and collaborative interactions. In: 2015 IEEE International Conference on Robotics and Automation (ICRA). pp. 624 - 629. DOI:10.1109/ICRA.2015.7139244, 2015.

[17] Minato T, Yoshikawa Y, Noda T, Ikemoto S, Ishiguro H and Asada, Cb2: A child robot with biomimetic body for cognitive developmental robotics. In: 2007 7th IEEE-RAS International Conference on Humanoid Robots. pp. 557 562. DOI:10.1109/ICHR.2007.4813926, 2007.

[18] De Maria G, Natale C and Pirozzi S, Force/tactile sensor for robotic applications. Sensors and Actuators A: Physical 175: 60 - 72, 2012.

[19] Arnaldo G. et al, Polymer optical fiber strain gauge for human-robot interaction forces assessment on an active knee orthosis, Optical Fiber technology 41, 205-211 (2018).

[20] Wang H, Jiang L, Xiang P, Improving the durability of the optical fiber sensor based on strain transfer analysis, Optical Fiber technology 42, 97-104 (2018).

[21] Iwata $\mathrm{H}$ and Sugano S, Whole-body covering tactile interface for human robot coordination. In: Proceedings 2002 IEEE International Conference on Robotics and Automation (Cat. No.02CH37292), volume 4. pp. 3818-3824 vol.4. DOI:10.1109/ROBOT.2002.1014315, 2002.
[22] Maggiali M, Cannata G, Maiolino P, Metta G, Randazzo M and Sandini G, Embedded distributed capacitive tactile sensor. In: 11th Mechatronics Forum Biennial International Conference, 2008.

[23] Cannata G, Maggiali M, Metta G and Sandini G, An embedded artificial skin for humanoid robots. In: 2008 IEEE International Conference on Multisensor Fusion and Integration for Intelligent Systems. pp. $434-438$, 2008.

[24] Mittendorfer P and Cheng G, Humanoid multimodal tactile-sensing modules. IEEE Transactions on Robotics 27(3): 401 - 410, 2011.

[25] Optoforce. https://optoforce.com/ 